\title{
Persistent cloaca: persistence of the challenge
}

\author{
Amr Abdelhamid AbouZeid*iD, Ahmed Bassiouny Radwan, Mohamed Eldebeiky and Sameh Abdel Hay
}

\begin{abstract}
Background: Persistent cloaca or cloacal anomalies represent a special category of anorectal anomalies affecting the female sex with a reported incidence of about one in 25,000 live birth.

The study included 34 cases of cloaca that were managed at our unit between 2003 through 2017. We retrospectively reviewed patients' records that included clinical presentation, investigations, operative data, and follow-up notes.

Anatomically, we stratified cloaca into three types according to the level of urogenital confluence. A low confluence (type 1) was defined by being at or below the level of the lower border of pubic symphysis with a short common channel (11 cases). A high confluence (type 3) was defined by being at or above the level of the upper border of pubic symphysis (9 cases). Between the low and high types, we defined an intermediate type (type 2) where the urogenital confluence was behind the mid-portion of pubic symphysis (14 cases).
\end{abstract}

Results: Renal anomalies were common association: solitary kidney in seven, pelvic kidney in two, and urinary tract dilatation (hydroureteronephrosis) in 12 cases. At follow-up, chronic renal insufficiency was detected in seven cases

The prognosis for urinary continence was excellent in low confluence (type 1) cloaca. On the other hand, urinary incontinence was common among type 3 (high confluence) cloaca (62.5\%).

Conclusion: Renal anomalies represent a common association with cloaca and a major cause of morbidity. Efforts should be directed to preserve renal function during the initial management, and to preserve the continence potential following the definitive repair.

Level of evidence: This is a case series with no comparison group (level IV).

Keywords: Cloaca, MRI, Surgery, Outcome, Classification

\section{Background}

Persistent cloaca or cloacal anomalies represent a special category of anorectal anomalies (ARA) affecting the female sex with a reported incidence of about one in 25,000 live birth [1]. The combination of urogenital and anorectal anomalies in the same subject complicates the clinical picture that it may be considered one of the most challenging situations in paediatric surgery $[2,3]$.

Searching through the literature, one can find the detailed descriptions and illustrations of Hardy

\footnotetext{
* Correspondence: amrabdelhamid@hotmail.com

Pediatric Surgery Department, Faculty of Medicine, Ain-Shams University,

Lotefy El-Sayed street, 9 Ain-Shams University buildings, Abbassia, Cairo 11657, Egypt
}

Hendren reporting his surgical experience in the management of a wide spectrum of complex cases of cloaca $[4,5]$. The introduction of the posterior sagittal approach gained wide-spread acceptance and increased our understanding about the surgical anatomy of the anal sphincters [6]. This has been reflected on improving the outcome of repairing the anorectal component of the anomaly. However, separation of the vagina from the common urogenital sinus remained a real challenge. The concept of urogenital sinus mobilization then appeared [7] aiming to save operative time and blood loss that may result from a difficult vaginal separation [3]; but still the technique 
has its limitations that it cannot be applied in all situations [8].

Here, we are reporting our experience in the management of a group of patients with cloaca over the last 15 years. Studying the surgical outcomes in cloaca was quite challenging for the rarity of cases being operated over a long period of time with multiple operating surgeons and evolution of surgical techniques. However, we have tried to highlight the difficulties that we have faced during management of these cases, and the lessons we have learned to overcome these difficulties.

\section{Methods}

The study included cases of cloaca that were managed at our unit between 2003 through 2017. We excluded cases of posterior cloaca from this study as they represent a separate entity (with different plan of management). We retrospectively reviewed patients' records that included clinical presentation, investigations, operative data, and follow-up notes. Available data included DICOM images for investigations (contrast studies, MRI), recorded videos of endoscopy, and operative photos.

Usually the diagnosis was made in the neonatal period based on the external appearance (female with imperforate anus and a single opening in the perineum; Fig. 1). In few cases, the diagnosis was suspected ante-Nataly (presence of hydrocolpos), while one case presented later during infancy at the age of 6 months. Faecal diversion (colostomy) was performed initially, while the definitive 'corrective' surgery was delayed after the age of 3 months. Decompression of the urogenital tract was required in eight cases (vaginostomy and/or vesicostomy; five and four cases respectively).

It may be generally accepted to classify cloaca according to the length of the common channel into either short or long channel cloaca: the longer the common channel, the higher the confluence $[9,10]$. However, we have found it difficult to apply the proposed cut-off value of $3 \mathrm{~cm}$ to differentiate between the two types (short or long) [11] for the following reasons:

- It does not consider the age variation at time of repair.

- Applying measurements in preoperative contrast X-ray studies is liable for magnification errors [12].

- In infancy, both the urethra and the common channel are fine structures showing narrow variation in length that should be perceived in millimetres. Approximating the measurements to centimetres during endoscopy may be rather subjective and nonreproducible.

We have tried to overcome some of the abovementioned limitations concerning the way of measuring the common channel and urethra by using MRI routinely in the preoperative assessment of cases of cloaca. However, discussing these diagnostic challenges is beyond our scope in this report and has been studied extensively in a previous one [13].

Anatomically, we stratified cloaca into three types according to the level of urogenital confluence in relation to the pubic symphysis (Fig. 2). This was defined preoperatively by MRI [13] and confirmed at cystoscopy. A low confluence (type 1) is defined by being at or below

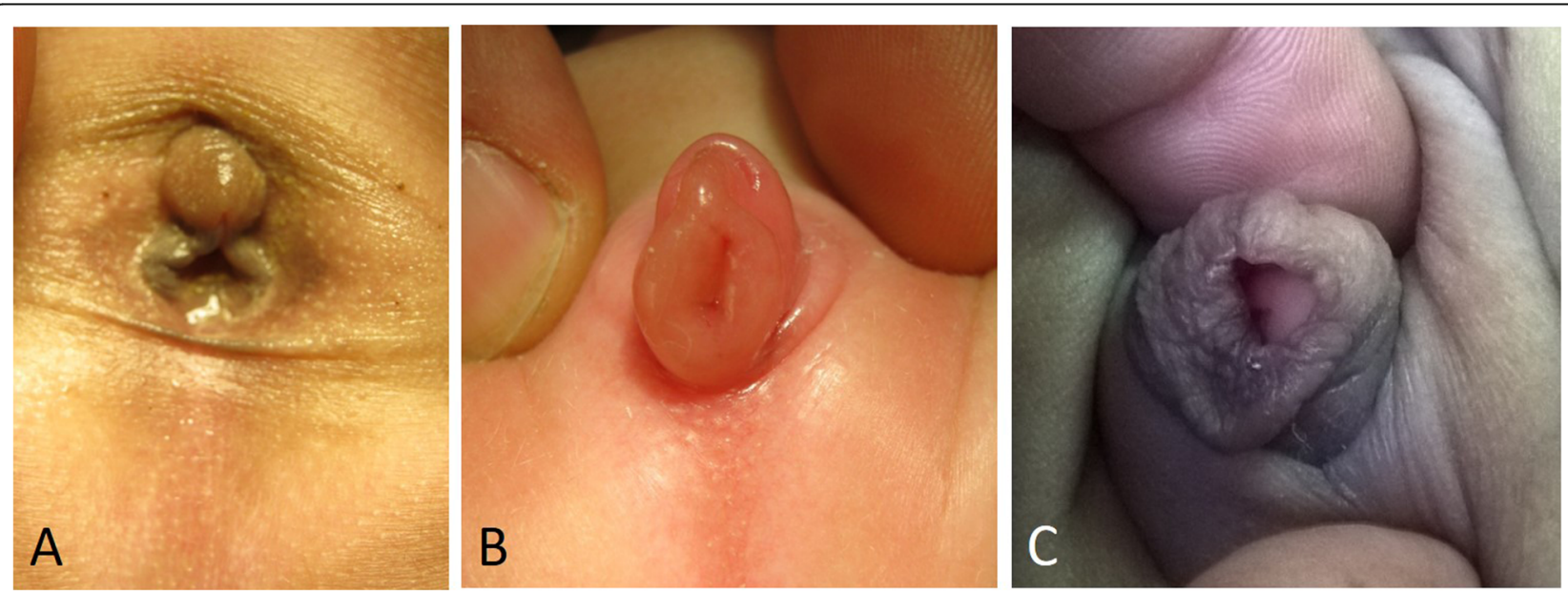

Fig. 1 The external appearance (female with imperforate anus and a single opening in the perineum) in 3 different cases of cloaca. a Hypoplastic external genitalia. b, c Abnormal skin protrusion around the common perineal orifice. Note: the external appearance in $\mathbf{b}$ and $\mathbf{c}$ was associated with a long common channel (high type) 


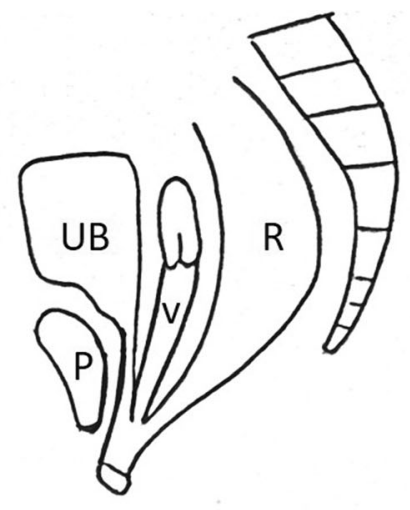

Type 1

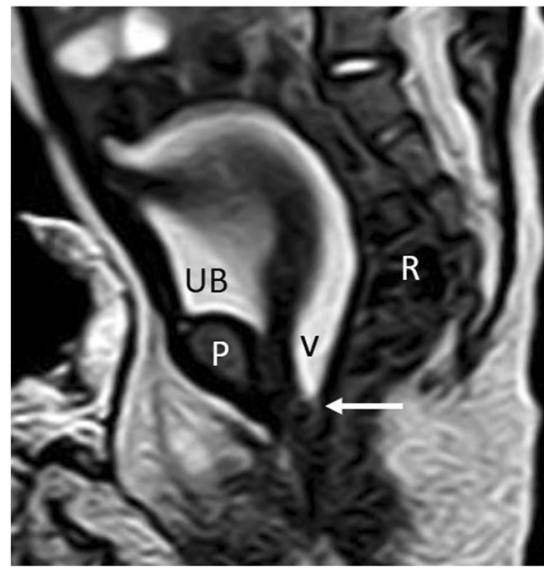

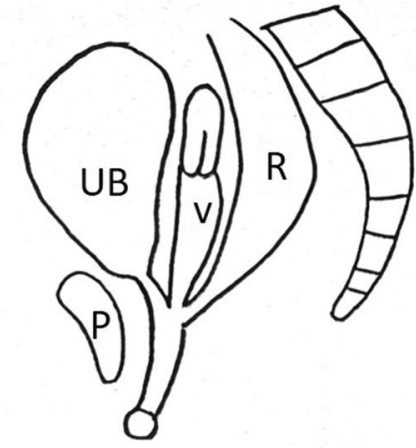

Type 2

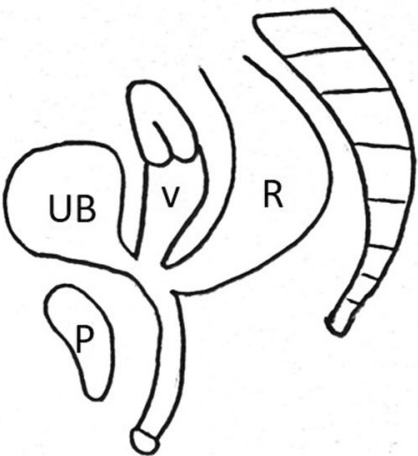

Type 3
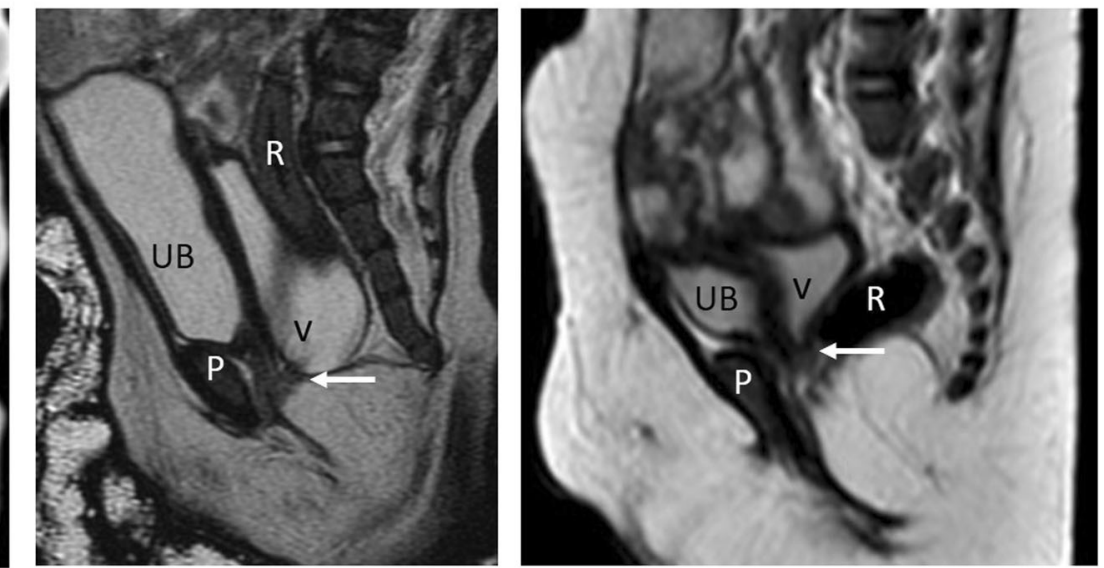

Fig. 2 New classification proposed for stratifying cloaca anatomically according to the level of urogenital confluence in relation to the pubic symphysis. The upper row is a diagrammatic representation for the new classification, while the lower row represents real MRI images for cases of cloaca (mid-sagittal T2WI). P, pubic symphysis; UB, urinary bladder; $v$, vagina; $R$, rectum; the transverse white arrow in the lower row points to the lower end of the vagina that corresponds to the level of urogenital confluence. Type 1 (low confluence), urogenital confluence at or below the level of the lower border of pubic symphysis (P); type 2 (intermediate), urogenital confluence at the level of the mid-portion of pubic symphysis; type 3 (high confluence), urogenital confluence at or above the level of the upper border of pubic symphysis

the level of the lower border of pubic symphysis with a short common channel (about $1 \mathrm{~cm}$ ). A high confluence (type 3 ) is close to the bladder neck (few $\mathrm{mm}$ ) and is defined by being at or above the level of the upper border of pubic symphysis. Between the low and high types, we defined an intermediate type (type 2 ) where the urogenital confluence was behind the mid-portion of pubic symphysis [13].

\section{Results}

The study included 34 cases of cloaca. Mean followup ranged from 1 to 15 years (mean 6.8 years, median 6.5 years). Unfortunately, we had two mortalities in this case series: one case died before definitive repair because of complications of associated cardiac anomalies, and another case died during operation (anaesthetic problem during a lengthy reconstructive procedure).

Renal anomalies were common association: solitary kidney in seven (20.6\%), pelvic kidney in two, and urinary tract dilatation (hydroureteronephrosis) in 12 cases (37.5\%). At follow-up, chronic renal insufficiency (persistent elevated serum creatinine) was detected in seven cases (four of them had a single kidney). Associated spinal anomalies included caudal regression (high spinal cord termination) in one, tethered cord in three cases, and another case of syringomyelia.

During the period of the study, there has been evolution of the surgical techniques starting by the posterior sagittal ano-rectovaginoplasty (PSARVP), partial/total urogenital sinus mobilization (PUM/TUM), abdominal/ 
Table 1 Procedures used for anorectoplasty in cases of cloaca, and the related functional outcome (bowel control)

\begin{tabular}{|c|c|c|c|c|c|}
\hline Procedure for anorectoplasty & $\begin{array}{l}\text { Number of } \\
\text { cases }\end{array}$ & $\begin{array}{l}\text { Voluntary bowel } \\
\text { control }\end{array}$ & $\begin{array}{l}\text { Regular laxatives } \\
\text { for evacuation }\end{array}$ & Regular enemas & $\begin{array}{l}\text { Less than } 3 \text { years or } \\
\text { lost to follow-up }\end{array}$ \\
\hline Perineal 'sagittal' anorectoplasty & 18 & $6(33.3 \%)$ & $3(16.6 \%)$ & $3(16.6 \%)$ & $6(33.3 \%)$ \\
\hline Abdomino-perineal pull-through & 10 & $2(20 \%)$ & $2(20 \%)$ & $\begin{array}{l}4(40 \%) \\
{[1 \text { caudal regression }+1 \text { tethered cord }]}\end{array}$ & $2(20 \%)$ \\
\hline Laparoscopic assisted & 4 & $1(25 \%)$ & -- & $\begin{array}{l}1(25 \%) \\
\text { (poor sacrum) }\end{array}$ & $2(50 \%)$ \\
\hline Total number & 32 & 9 (28.1\%) & $5(15.6 \%)$ & $8(25 \%)$ & 10 (31.3\%) \\
\hline
\end{tabular}

laparoscopic-assisted rectal pull-through, vaginal pullthrough, and other advanced vaginoplasties (ileo/colovaginoplasty). The evolution of the surgical technique also included staging of the corrective surgery in certain cases to decrease the surgical stress and hazards of lengthy reconstructive operations in infancy. This has been encouraged after the introduction of laparoscopy, where the anorectoplasty is performed first, to be followed by the repair of the common urogenital sinus at a second stage. Tables 1 and 2 summarize the reconstructive procedures performed during the period of the study and their related functional outcome at follow-up. Figures 3, 4, 5, and 6) illustrate the examples of the different surgical approaches used for the repair of common urogenital sinus abnormality depending on the level of the urogenital confluence (low, intermediate, and high confluence).

The prognosis for urinary continence was excellent in low confluence (type 1) cloaca; all except one (with caudal regression) were continent (spontaneous voiding). On the other hand, urinary incontinence (usually in the form of poor evacuation of the bladder) was common among type 3 (high confluence) cloaca. Urinary incontinence was $62 \%$ among type 3 compared with $14 \%$ and $10 \%$ among type 2 and type 1, respectively. Table 3 summarizes the functional outcome (urinary continence) in relation to the type of cloaca based on the level of urogenital confluence. Regarding the operative technique, urinary incontinence was high following vaginal pull-through and ileo/colo-vaginoplasties $(75 \%$ and $50 \%$ respectively) compared with other perineal approaches (Table 2).

Disorders of bowel control were managed either by laxatives or enemas through bowel management program at the incontinence clinic (Table 1). Urinary incontinence was often more distressing and required different surgical procedures: Mitrofanoff procedure for clean intermittent catheterization (3 cases), bladder augmentation (1 case), and continent urinary diversion (3 cases).

\section{Discussion}

Persistent cloaca comprises two principle abnormalities namely the anorectal anomaly and the common urogenital sinus, which are not necessarily affected to the same extent in the same subject [10]. A low urogenital confluence may be associated with a high rectum [9] and vice versa. Therefore, it may be more practical to discuss (plan) the management of each component separately.

In contrast to the urogenital abnormality, the management of the anorectal component of cloaca is usually more straightforward. Mobilization of the rectum is relatively easier based on its robust submucosal

Table 2 Procedures used for correction of common urogenital sinus in cases of cloaca and the related functional outcome (urinary control)

\begin{tabular}{lllll}
\hline Procedure & Number & Voiding spontaneously & Urinary incontinence & Less than 3 years or lost to follow-up \\
\hline Interoitoplasty & 6 & $2(33.3 \%)$ & $\begin{array}{l}1(16.6 \%) \\
\text { (caudal regression) }\end{array}$ & $3(50 \%)$ \\
PUM & 9 & $7(77.8 \%)$ & -- & $2(22.2 \%)$ \\
TUM & 7 & $1(14.3 \%)$ & $2(28.6 \%)$ & $4(57.1 \%)$ \\
PSARVP & 2 & $1(50 \%)$ & -- & $1(50 \%)$ \\
Vaginal pull-through & 4 & $1(25 \%)$ & $3(75 \%)$ & -- \\
lleo/colo-vaginoplasty & 4 & $2(50 \%)$ & $2(50 \%)$ & -- \\
Total & 32 & $14(43.7 \%)$ & $(1$ poor sacrum + 1 tethered cord) & $10(31.3 \%)$ \\
\hline
\end{tabular}

PUM partial urogenital sinus mobilization, TUM total urogenital sinus mobilization, PSARVP posterior sagittal anorectovagino-plasty 

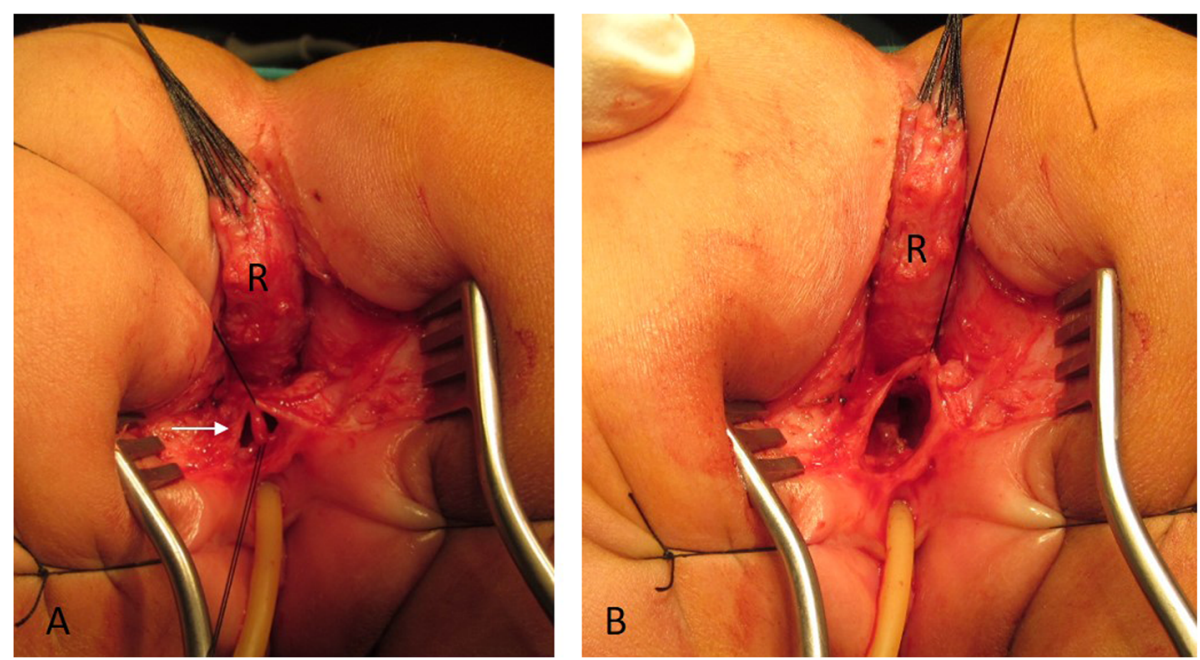

Fig. 3 Type 1 (low urogenital confluence) cloaca. The rectum (R) is separated and mobilized from the urogenital tract. A urinary catheter is seen inside the urethra at the bottom (patient is in prone position). a Septated vagina (arrow) located between rectum and urethra. b Partial mobilization of the vagina by limited dissection on dorsal and lateral aspects without extending the dissection to the urethra; note: the vaginal septum has been divided

blood supply. A perineal (posterior sagittal) approach is usually sufficient for the mobilization of the rectum reaching below the mid-sacral piece (S3); otherwise, abdominal or laparoscopic assistance may turn to be necessary [10]. On the other hand, vaginal reconstruction is often the most challenging step in cloaca [3]. This is because of the difficulty frequently encountered during vaginal mobilization due to its intimate adherence to the urethra and unreliable submucosal blood supply $[3,9,10,14]$.
At both ends of the spectrum, the management of persistent urogenital sinus appears to be less controversial. A low confluence is managed by PUM (or even just introitoplasty) with excellent functional and cosmetic outcome $[9,10]$. On the other end of the spectrum, a high confluence is managed by keeping the common channel to act as a urethra, while the vagina is separated and brought down to the perineum (if its length allows for a direct vaginal pullthrough); otherwise, a piece of bowel is used to

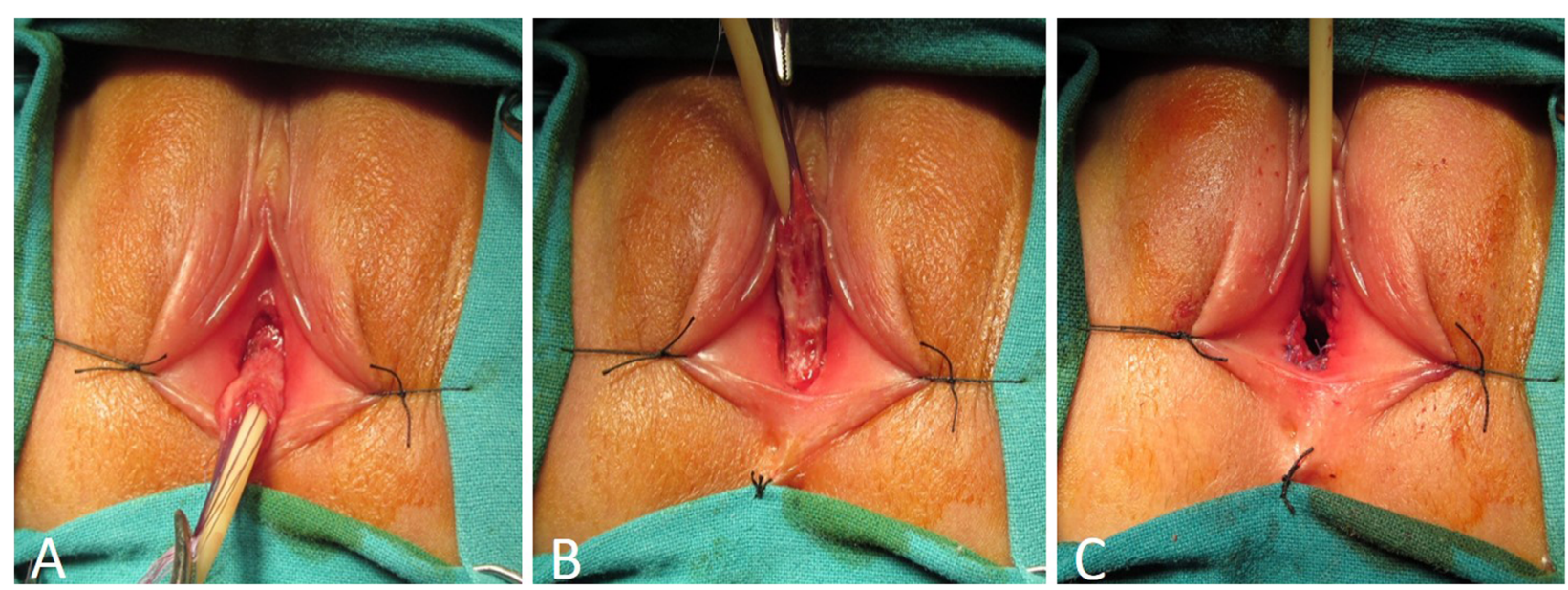

Fig. 4 Type 1 (low urogenital confluence) cloaca. Partial urogenital sinus mobilization with the patient in the supine position (note: the anorectoplasty was done at a previous separate stage): $\mathbf{a}$, b Dissection and mobilization of the common urogenital sinus. c Suturing the vagina and urethra to the vestibule after excision of the short common sinus 

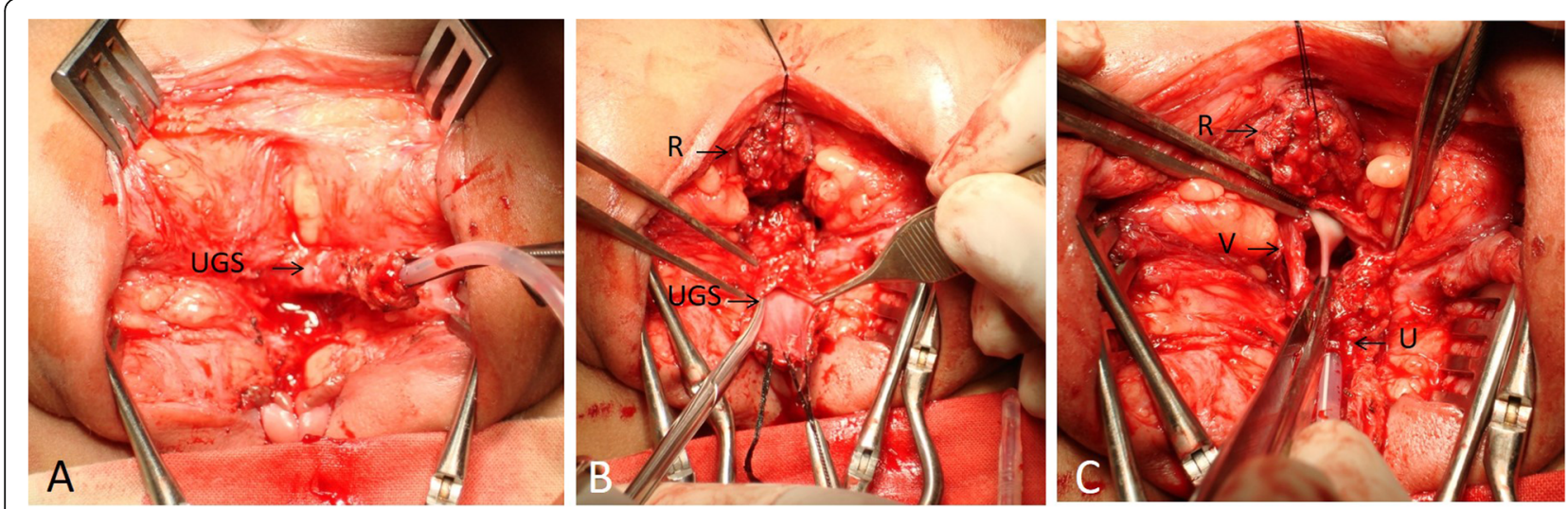

Fig. 5 Type 2 (intermediate urogenital confluence) cloaca. Total urogenital sinus mobilization (TUM procedure): a With the patient in the prone position, dissection, and mobilization of the common urogenital sinus (UGS). $\mathbf{b}$ The rectum (R) is separated and mobilized from the urogenital tract, incision through the mobilized urogenital sinus (UGS) to expose the vaginal introitus. $\mathbf{c}$ The vagina $(V)$ is seen with a longitudinal septum, and a catheter is seen within the urethra $(U)$

traverse the gap down to the perineum (ileo/colo-vaginoplasty) [10].

The separation of the vagina from the urinary tract seems to be most difficult in the intermediate types of cloaca. Separation is difficult from below (perineal posterior sagittal approach) as well as from the abdomen [3]. The concept of urogenital sinus mobilization has been proposed to overcome this difficult separation [7]; however, the results are not always reproducible $[9,10$, 15]. Partial urogenital sinus mobilization is not suitable for all cases and may end with a high vagina. Total urogenital sinus mobilization is more liable for tissue ischaemia especially with a combined abdomino-perineal dissection [8], and still its long-term effect on the continence mechanism is questionable $[3,16,17]$.

In this report, we presented an overview on the surgical management of a rare disease in paediatric surgery. The report also included the outcome of the different surgical procedures, while referring to a new proposal for stratifying cloaca [13]. We did not assess gynaecological and sexual aspects as these will need longer follow-up and involvement of other specialities as well.
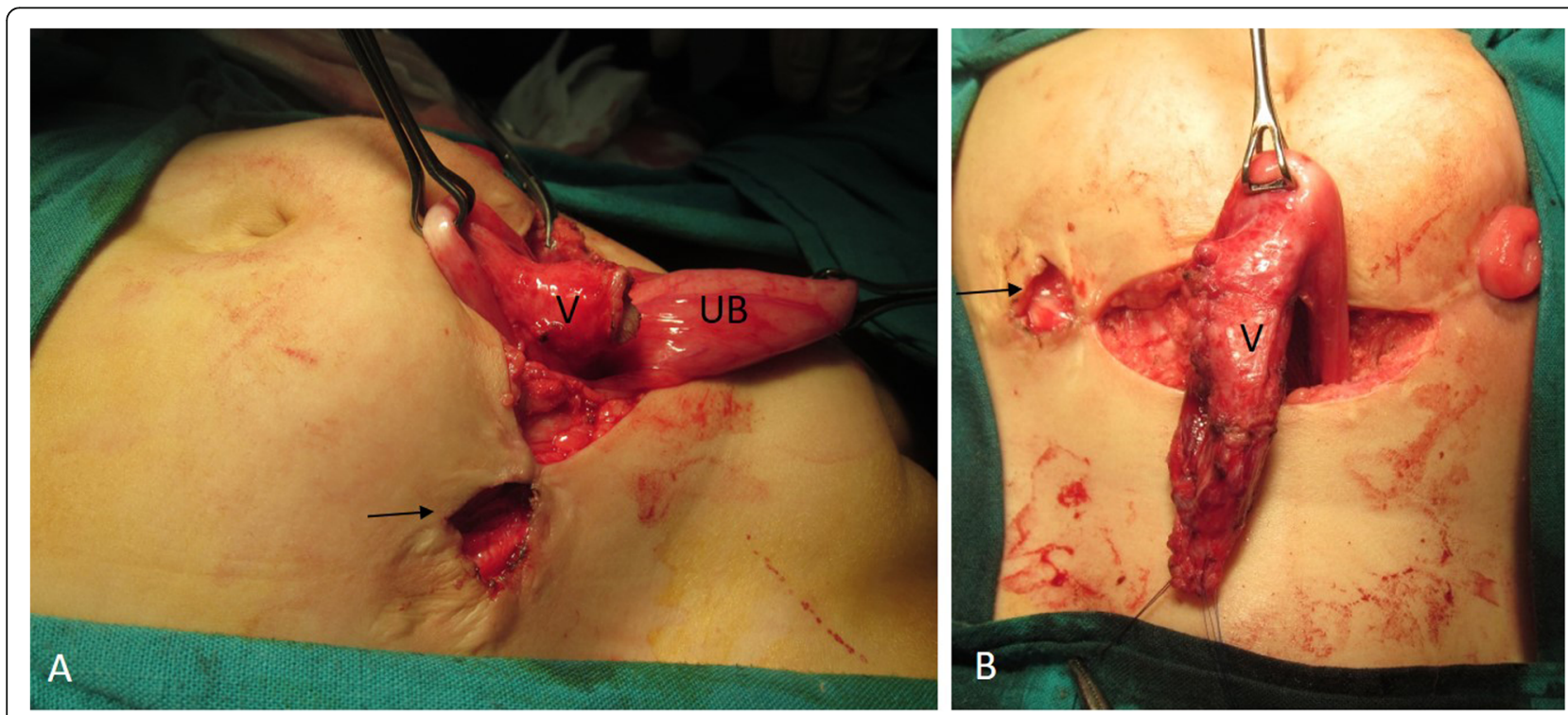

Fig. 6 Type 3 (high urogenital confluence) cloaca. The patient was initially managed in the neonatal period by a colostomy and a vaginostomy (black arrow points to site of vaginostomy on the skin). Vaginal pull-through: a the vaginostomy $(V)$ is detached from the abdominal wall (UB, urinary bladder). $\mathbf{b}$ The vagina is separated from the back of the bladder and reconstructed to form a tube that is ready for a pull-through procedure 
Table 3 Correlating the functional outcome (urinary control) to the type of cloaca based on the level of urogenital confluence

\begin{tabular}{lllll}
\hline Urogenital confluence & Number & Voiding spontaneously & Urinary incontinence & Less than 3 years or lost to follow-up \\
\hline Low confluence (type 1) & 10 & $5(50 \%)$ & $\begin{array}{l}1(10 \%) \\
\text { (caudal regression) }\end{array}$ & $4(40 \%)$ \\
Intermediate confluence (type 2) & 14 & $7(50 \%)$ & $2(14.3 \%)$ & $5(35.7 \%)$ \\
High confluence (type 3) & 8 & $2(25 \%)$ & $\begin{array}{l}5(62.5 \%) \\
(1 \text { poor sacrum + 1 tethered cord) }\end{array}$ & $1(12.5 \%)$ \\
Total & 32 & $14(43.7 \%)$ & $8(25 \%)$ & $10(31.3 \%)$ \\
\hline
\end{tabular}

Another limitation was the subjective evaluation for continence, as objective assessment scores were not available. We believe the outcome is still less satisfactory even in highly specialized centres all over the world [1]. The great diversity and complexity of the cloacal spectrum may present an obstacle for a unified classification system $[11,13]$. The collaboration between different centres and specialities is needed to provide more suitable surgical solutions based on better understanding of the complex anatomy of the anomaly, which in turn can help to improve the outcome.

\section{Conclusion}

Renal anomalies represent a common association with cloaca and a major cause for morbidity. Efforts should be directed to preserve renal function during the initial management and to preserve the continence potential following the definitive repair.

\section{Abbreviations}

ARA: Anorectal anomalies; PSARVP: Posterior sagittal ano-rectovaginoplasty; PUM: Partial urogenital sinus mobilization; TUM: Total urogenital sinus mobilization

\section{Acknowledgements}

None

\section{Authors' contributions}

AAAZ conceived of the study and participated in its design. AAAZ and ABR participated in data acquisition. AAAZ and $A B R$ participated in the analysis and interpretation of data. AAAZ drafted the manuscript. ME and SAH performed critical revision. All authors read and approved the final manuscript.

\section{Funding}

None

\section{Availability of data and materials}

The datasets used and/or analysed during the current study are available from the corresponding author on reasonable request.

\section{Ethics approval and consent to participate}

A written consent was taken before operation in all cases. Owing to the retrospective nature of the study, an IRB number was not required, and the study was approved through expedited review by the scientific/ethical committee of the Surgery Department (Faculty of Medicine, Ain-Shams University).

\section{Consent for publication}

Patient identity did not appear in any part of the manuscript; therefore, consent for publication was not required.

\section{Competing interests}

The authors declare that they have no competing interest.

Received: 1 October 2019 Accepted: 12 November 2019

Published online: 03 January 2020

\section{References}

1. Versteegh HP, van Rooij IA, Levitt MA, Sloots CE, Wijnen RM, de Blaauw I. Long-term follow-up of functional outcome in patients with a cloacal malformation: a systematic review. J Pediatr Surg. 2013;48:2343-50.

2. Rink RC, Herndon CDA, Cain MR, Kaffer M, Dussinger AM, King SJ, Casale AJ. Upper and lower urinary tract outcome after surgical repair of cloacal malformations: a three-decade experience. BJU Int. 2005;96:131-4

3. Leclair MD, Gundetti M, Kiely EM, Wilcox DT. The surgical outcome of total urogenital mobilization for cloacal repair. J Urol. 2007;177:1492-5.

4. Hendren H. Urogenital sinus and anorectal malformations: experience with 22 cases. J Pediatr Surg. 1980;15:628-41.

5. Hendren $\mathrm{H}$. Cloacal malformations: experience with 105 cases. J Pediatr Surg. 1992;27:890-901.

6. DeVries PA, Pena A. Posterior sagittal anorectoplasty. J Pediatr Surg. 1982;17: 638-43.

7. Pena A. Total urogenital mobilization - an easier way to repair cloacas. J Pediatr Surg. 1997:32:263-7.

8. Wood RJ, Reck-Burneo CA, Dajusta D, Ching C, Jayanthi R, Bates DG, et al. Cloaca reconstruction: a new algorithm which considers the role of urethral length in determining surgical planning. J Pediatr Surg. 2018;53:90-5.

9. Levitt MA, Pena A. Cloacal malformations: lessons learned from 490 cases. Semin Pediatr Surg. 2010;19:128-38.

10. Bischoff A. The surgical treatment of cloaca. Semin Pediatr Surg. 2016;25: 102-7.

11. Caldwell BT, Wilcox DT. Long-term urological outcomes in cloacal anomalies. Semin Pediatr Surg. 2016;25:108-11.

12. Patel MN. Use of rotational fluoroscopy and 3-D reconstruction for preoperative imaging of complex cloacal malformations. Semin Pediatr Surg. 2016;25:96-101.

13. AbouZeid AA, Mohammad SA. The cloacal anomalies: anatomical insights through a complex spectrum. J Pediatr Surg. In press, corrected proof, Available online 18 April 2019; https://doi.org/https://doi.org/10.1016/j. jpedsurg.2019.04.005

14. Bischoff A, Levitt MA, Breech L, Hall J, Pena A. Vaginal switch—a useful technical alternative to vaginal replacement for select cases of cloaca and urogenital sinus. J Pediatr Surg. 2013;48:363-6.

15. Levitt MA, Bischoff A, Pena A. Pitfalls and challenges of cloaca repair: how to reduce the need for reoperations. J Pediatr Surg. 2011:46:1250-5.

16. Palmer BW, Trojan B, Griffin K, Reiner W, Wisniewski A, Frimberger D, Kropp BP. Total and partial urogenital mobilization: focus on urinary continence. J Urol. 2012;187:1422-6.

17. AbouZeid AA. Achieving full anatomical correction in girls with cloacal anomalies: a necessity or an overdoing? J Genital Surg 2019; DOI: https:// doi.org/10.21608/JGS.2019.7646.1015

\section{Publisher's Note}

Springer Nature remains neutral with regard to jurisdictional claims in published maps and institutional affiliations. 